\title{
A Nine-year Follow-up of a Porcelain Laminate Veneer Restoration Applied to an Adolescent with Traumatized Dentition: A Clinical Report
}

\author{
Naomi Tanoue, DDS, PhD, ${ }^{a}$ Mitsuru Atsuta, DDS, PhD, ${ }^{b}$ and Hideo Matsumura, DDS, $\mathrm{PhD}^{\mathrm{c}}$ \\ ${ }^{a}$ Department of Aesthetic Dentistry, Nagasaki University Hospital of Medicine and Dentistry, Nagasaki, Japan \\ ${ }^{\mathrm{b}}$ Division of Fixed Prosthodontics and Oral Rehabilitation, Nagasaki University Graduate School of Biomedical \\ Sciences, Nagasaki, Japan \\ ${ }^{\mathrm{c}}$ Department of Fixed Prosthodontics, Nihon University School of Dentistry, Tokyo, Japan
}

\section{Clinical significance}

The clinical course here suggests the importance of selecting a method compatible with a young patient for masking the discoloration of a permanent anterior tooth.

\begin{abstract}
Patients: A 9-year-old Japanese female patient with an unremarkable medical history was initially $\mathrm{ex}^{-}$ amined for the chief complaint of slight pain and discomfort in a traumatized maxillary central incisor. Four years after endodontic treatment, the patient complained of dark discoloration of the tooth. Among the treatments/restorations indicated, the patient and her parent consequently selected and approved of a porcelain laminate veneer restoration because of its advantage of minimal tooth reduction. The maxillary diastema was gradually closed after insertion of a porcelain laminate veneer, and 9 years of follow-up have revealed that the prosthodontic treatment successfully restored the tooth.

Discussion: Many methods have been introduced as aesthetic solutions to discoloration. Although the patient was satisfied with the prosthodontic approach from a functional as well as an aesthetic point of view, direct composite restorations might be an alternative for younger patients in mixed dentition.

Conclusion: The treatment in a growing individual should be carefully planned and performed, considering the development of permanent dentition.
\end{abstract}

Key words: devitalization, diastema, discoloration, laminate veneer, porcelain

\section{Corresponding to: Dr Naomi Tanoue}

Department of Aesthetic Dentistry, Nagasaki University

Hospital of Medicine and Dentistry

1-7-1 Sakamoto, Nagasaki 852-8501, Japan

Tel: +81-95-849-7688, Fax: +81-95-849-7689

E-mail: t-naomi@net.nagasaki-u.ac.jp

Received on October 12, 2006 / Accepted on December 20, 2006

\section{Introduction}

Children frequently experience trauma to the anterior teeth. The traumatized teeth are occasionally compromised by devitalization and thereafter, by dark coronal discoloration. The discoloration of anterior teeth is an aesthetic concern, even in children. In particular, in this case involving the permanent maxillary central incisor in a young girl, both the child and her parents/guardians were eager to repair the damage.

Many methods have been introduced as aesthetic solutions to discoloration. Direct composite restorations, including a composite layering technique, have been reported to contribute in improving the aesthetics and functions of discolored anterior teeth. ${ }^{1}$ Poyser et $\mathrm{al}^{2}$ reported the first technical account of the application of the inside/ outside bleaching technique to discolored teeth. Lynch and $\mathrm{McConell}^{3}$ indicated that enamel microabrasion technique was useful in removing discolored and/or pitted enamel. Irrespective of the method used, treatments for a growing individual should be carefully performed considering the development of permanent dentition.

In this case report, a trial had been undertaken of the use of a prosthodontic system for masking discoloration; a laminate veneer system, which requires minimal tooth reduction, enables accurate replication of the complex internal structures, visible shape, color, and surface anatomy of natural teeth. ${ }^{4-10}$ This paper presents the results of a prosthodontic approach using the porcelain laminate veneer system for the treatment of a discolored anterior tooth in the permanent dentition of a child. 


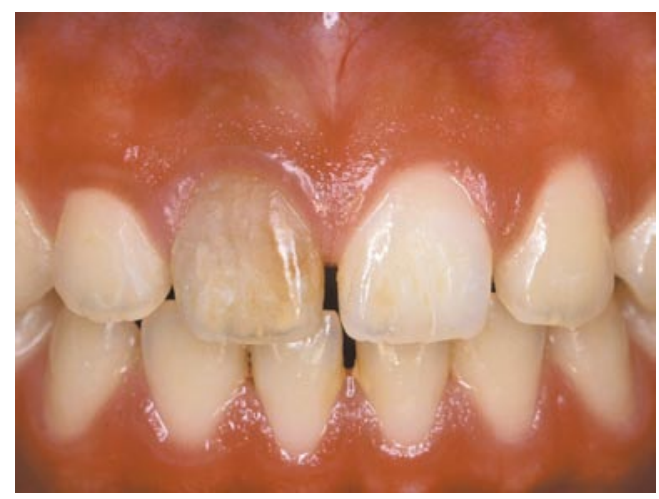

Fig. 1 Pre-treatment view of the traumatized maxillary central incisor.

\section{Outline of the case}

A 9-year-old Japanese female patient with an unremarkable medical history was initially examined at our university hospital for the chief complaint of slight pain and discomfort in a traumatized maxillary central incisor. Clinical examination revealed insignificant tooth mobility. Splinting and endodontic therapy were used for the treatment. Once the symptoms subsided, the splint was removed and the conventional cavity of the lingual surface was filled with a composite resin material (Clearfil APX, A3, Kuraray Medical Inc, Tokyo, Japan) for endodontic treatment.

Four years after the filling, the patient complained about dark discoloration of the tooth (Fig. 1). Although the application of the intracoronal bleaching technique had been indicated initially to avoid tooth reduction, the patient opted for the treatment that could be completed in a short time period.

At the time of treatment, the patient was in incomplete permanent dentition and maxillary diastema existed. After considering the development of permanent dentition, prosthodontic treatment plans for accurate replication of the complex structure, visible shape, color, and surface anatomy of the natural tooth were proposed as alternative treatments. Among the prosthodontic restorations indicated, the patient and her parent consequently selected and approved of a porcelain laminate veneer restoration because of its advantage of minimal tooth reduction. They also understood that the treatment would not eliminate the existing diastema.

The facial surface of the incisor was prepared (Fig. 2). A $0.5-\mathrm{mm}$ tooth reduction was performed by creating a chamfer cervical finish line, us-

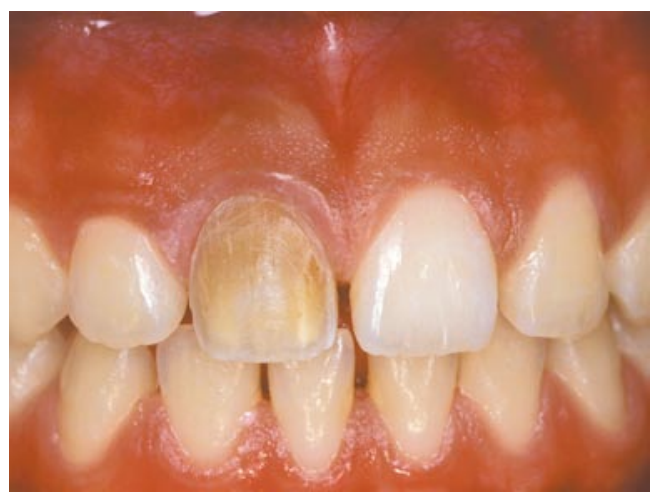

Fig. 2 Preparation for restoration with porcelain laminate veneer.

ing a high-speed rotary cutting instrument with diamond points (ISO 199/014 and 199/016, Shofu Inc., Kyoto, Japan) and a water coolant. All tooth preparations were completed without any sharp line angles. A shade was selected by using the Vita Lumin Vacuum Shade Guide (Vita Zahnfabrik, Bad Säckingen, Germany).

An impression of the entire maxilla was prepared with silicone elastomeric materials $\left(\mathrm{Ex}^{-}\right.$ afine Putty and Injection, GC Corp., Tokyo, Japan) by using a stock tray and poured on a die stone (Fuji Rock, GC Corp.). A facebow transfer was used to mount the maxillary cast onto a semi-adjustable articulator (Spacy Articulator, YDM Corp., Tokyo, Japan), and a centric relation record was registered using a vinyl polysiloxane impression material (Exabite II, GC Corp.) for mounting the opposing cast onto the articulator. Protrusive records were prepared with the impression material (Exabite II), and the sagittal and lateral condylar paths were adjusted on the articulator. A porcelain laminate veneer restoration was then fabricated to accurately simulate the appearance of the other central incisor; this was done by using a feldspathic porcelain system (Lamina and Vintage, Shofu Inc.) according to the manufacturer's directions.

After completion of its fabrication and adjustment, the laminate veneer was evaluated for its fit on the prepared tooth. The inner surface to be bonded was etched with 5\% hydrofluoric acid (HF-Gel, GC Corp.) for 60 seconds, ${ }^{11}$ washed with tap water, ultrasonically cleaned in methanol, lightly air-dried with an air syringe, and primed with three-liquid ceramic primer (Clapearl Bonding Agent, Kuraray Medical Inc.). ${ }^{12}$ The prepared enamel surface was etched with $40 \%$ phosphoric acid (K-Etchant, Kuraray Medical Inc.) for 30 


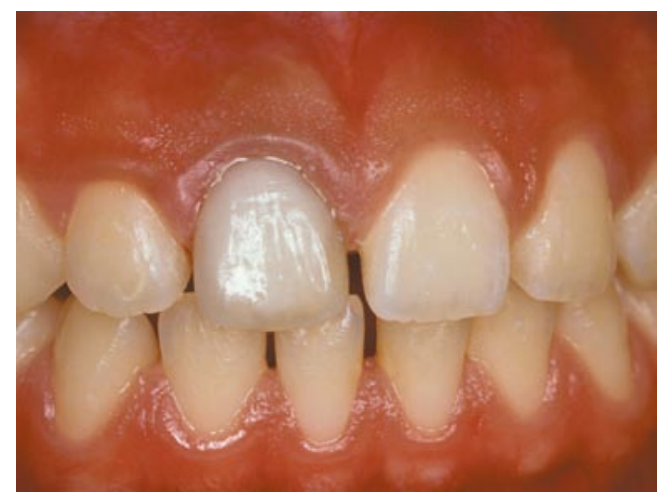

Fig. 3 Post-treatment view of the traumatized maxillary central incisor.

seconds, washed and air-dried with an air syringe, and primed with a bonding agent (Clearfil Photobond, Kuraray Medical Inc.). Thereafter, the laminate veneer was luted with a dual curable composite luting agent (Clapearl DC, Universal, Kuraray Medical Inc.). Photopolymerization was performed with a light-polymerizing unit (Quicklight, J. Morita Corp., Suita, Japan) for 60 seconds each for the incisal, mesial, and distal surfaces. Figure 3 shows the post-treatment view of the restoration.

Although the color of the treated tooth could not be completely harmonized with the patient's original teeth, she was satisfied with the treatment, both aesthetically and functionally. After the treatment, the patient was followed-up at 6 -month intervals for oral hygiene and evaluation of dentition. The maxillary midline diastema gradually closed by 5 years after insertion (Fig. 4 ), and the laminate veneer has been functioning well for more than 9 years of clinical service (Fig. $5)$.

\section{Discussion}

In this case, timely masking of the discoloration of a traumatically injured anterior tooth was imminent. For younger patients with mixed dentition, direct composite restorations may be feasible temporary alternatives for the patient's immediate needs. ${ }^{1}$ Spinas ${ }^{13}$ evaluated the longevity of composite restorations of traumatically injured teeth and concluded that composites could not be used for long-term restorations and that prosthodontic restorations are better alternatives. The laboratory-fabricated porcelain laminate veneer system requires minimal tooth

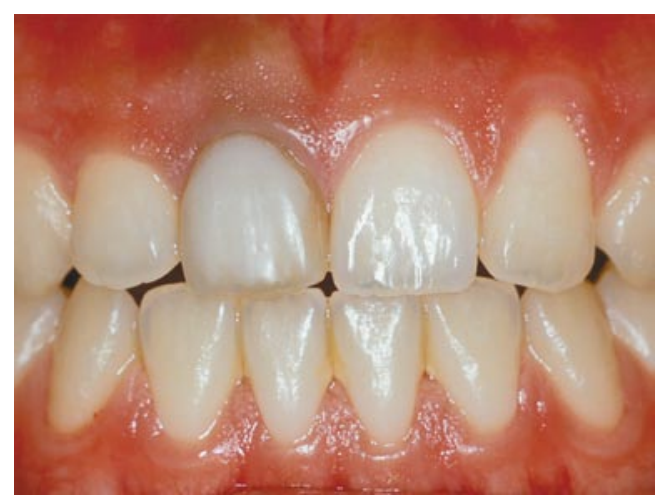

Fig. 4 Facial view 5 years after insertion. The midline diastema was closed.

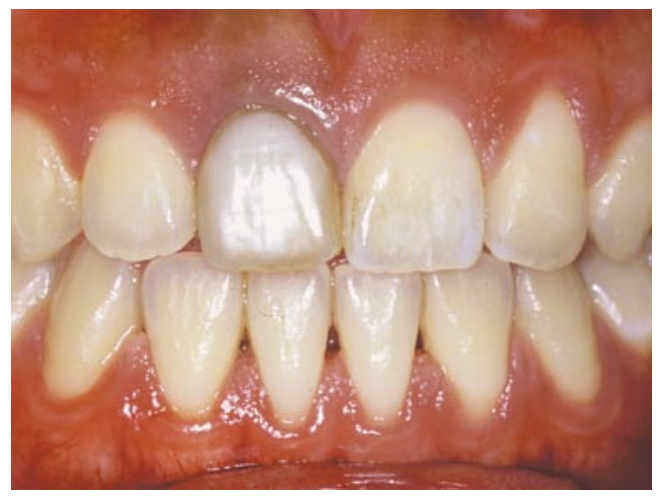

Fig. 5 Facial view 9 years after insertion.

reduction, and it is recognized as a long-term restoration technique to alter tooth position, shape, size, and color. Therefore, a porcelain laminate veneer was selected because of the patient's dentition stage and aesthetic need. The indirect veneer has been functioning for more than 9 years without any problems such as debonding and chipping, except for slight staining and exposure of a margin.

It is understood that prosthodontic approaches such as the use of the laminate veneer system might not always be the best method. In fact, the shade of the porcelain veneer fabricated for this case did not completely match the patient's own teeth even at the time of insertion. The inharmonious color might be due by the fact that the porcelain laminate veneer was very thin to conceal the dark, discolored background. However, considering the patient's age and incomplete development of permanent dentition at the time of treatment, the porcelain laminate veneer was believed to be appropriate from the standpoint of minimal intervention. If necessary, the laminate veneer could be replaced by other prosthodontic 
restorations in the future considering permanent dentition in the patient would have successfully erupted.

\section{Conclusion}

The method of treatment for children should be selected after considering factors such as anteroposterior relationship of the arches, position of the canines at the time of treatment, patient's age, dentition stage, and patient's aesthetic needs. With regard to a patient undergoing early permanent dentition, the continued eruption and positioning of clinical crowns with growth occasionally require that the indirect restoration be replaced several times to remain serviceable both aesthetically and functionally. The treatment in a growing individual should be carefully planned and executed, considering the development of permanent dentition.

\section{References}

1. Milnar FJ. Selecting nanotechnology-based composites using colorimetric and visual analysis for the restoration of anterior dentition: a case report. J Esthet Restor Dent 16: 89-100, 2004.

2. Poyser NJ, Kelleher MG, Briggs PF. Managing discoloured non-vital teeth: the inside/outside bleaching technique. Dent Update 31: 204-210, 213-214, 2004.

3. Lynch CD, McConnell RJ. The use of microabrasion to remove discolored enamel: a clinical report.
J Prosthet Dent 90: 417-419, 2003.

4. Velasco LF, de Araujo FB, Ferreira ES et al. Esthetic and functional treatment of a fused permanent tooth: a case report. Quintessence Int 28: 677-680, 1997.

5. Cehreli MC, Iplikcioglu H. Five-year follow-up of InCeram laminate restorations: a clinical report. J Prosthet Dent 84: 487-489, 2000.

6. Bergendal B. Prosthetic habilitation of a young patient with hypohidrotic ectodermal dysplasia and oligodontia: a case report of 20 years of treatment. Int J Prosthodont 14: 471-479, 2001.

7. Claman L, Alfaro MA, Mercado A. An interdisciplinary approach for improved esthetic results in the anterior maxilla. J Prosthet Dent 89: 1-5, 2003.

8. Sari T, Usumez A. Restoring function and esthetics in a patient with amelogenesis imperfecta: a clinical report. J Prosthet Dent 90: 522-525, 2003.

9. Okamura M, Chen KK, Kakigawa $\mathrm{H}$ et al. Application of alumina coping to porcelain laminate veneered crown: part 1 Masking ability for discolored teeth. Dent Mater J 23: 180-183, 2004.

10. Chen JH, Shi CX, Wang M et al. Clinical evaluation of 546 tetracycline-stained teeth treated with porcelain laminate veneers. J Dent 33: 3-8, 2005.

11. Chen JH, Matsumura H, Atsuta M. Effect of etchant, etching period, and silane priming on bond strength to porcelain of composite resin. Oper Dent 23: 250-257, 1998.

12. Matsumura H, Kato H, Atsuta M. Shear bond strength to feldspathic porcelain of two luting cements in combination with three surface treatments. J Prosthet Dent 78: 511-517, 1997.

13. Spinas E. Longevity of composite restorations of traumatically injured teeth. Am J Dent 17: 407-411, 2004. 